\title{
Association between abdominal obesity and liver steatosis and fibrosis among patients with chronic hepatitis B measured by Fibroscan
}

\author{
JING SUN $^{1 *}$, YANFANG LI ${ }^{1 *}$, XUYING SUN ${ }^{1}$, YOUDE LIU ${ }^{1}$, DANXIA ZHENG ${ }^{2}$ and LIJUAN FAN ${ }^{2}$ \\ ${ }^{1}$ Department of Hepatology, Yantai Infectious Disease Hospital, Yantai, Shandong 264000; \\ ${ }^{2}$ Department of Gastroenterology, Jining First People's Hospital, Jining, Shandong 272000, P.R. China
}

Received October 24, 2018; Accepted April 5, 2019

DOI: $10.3892 /$ etm.2019.7727

\begin{abstract}
The present cross-sectional study aimed to assess hepatic fibrosis in chronic hepatitis $\mathrm{B}(\mathrm{CHB})$ patients with abdominal obesity and to explore the associated indicators. A total of $615 \mathrm{CHB}$ patients were enrolled and 287 of them had abdominal obesity. The liver stiffness value was measured using Fibroscan. The diagnosis of liver fibrosis was confirmed by a liver stiffness value of $>7.4 \mathrm{kPa}$, and a value of $>10.6 \mathrm{kPa}$ was considered to indicate advanced liver fibrosis. The Fibroscan results suggested that the liver stiffness value in patients with abdominal obesity was significantly higher than that in patients without abdominal obesity $(9.94 \pm 11.59$ vs. $7.47 \pm 7.58 \mathrm{kPa} ; \mathrm{P}=0.002)$. The proportions of patients with liver fibrosis and advanced liver fibrosis among patients with abdominal obesity were significantly higher than those among patients without abdominal obesity $(\mathrm{P}=0.011)$. Multivariate logistic regression analysis indicated that a high aspartate aminotransferase (AST) level [odds ratio $(\mathrm{OR})=2.991 ; \mathrm{P}<0.001]$, smoking $(\mathrm{OR}=2.002 ; \mathrm{P}=0.019)$ and diabetes mellitus $(\mathrm{OR}=2.047 ; \mathrm{P}=0.029)$ were independent indicators for liver fibrosis in $\mathrm{CHB}$ patients with abdominal obesity. Furthermore, a high AST level (OR=1.024; $\mathrm{P}<0.001)$, alcohol consumption $(\mathrm{OR}=1.994 ; \mathrm{P}=0.032)$ and diabetes mellitus $(\mathrm{OR}=1.977 ; \mathrm{P}=0.045)$ were independent indicators for advanced hepatic fibrosis. The indicators associated with liver steatosis included high body weight $(\mathrm{OR}=1.113$; $\mathrm{P}<0.001)$ and high diastolic blood pressure $(\mathrm{OR}=1.079$; $\mathrm{P}=0.002$ ). In conclusion, the present study indicated that abdominal obesity significantly exacerbates liver fibrosis
\end{abstract}

Correspondence to: Dr Lijuan Fan, Department of Gastroenterology, Jining First People's Hospital, 6 Health Road, Jining, Shandong 272000, P.R. China

E-mail: fanlijuan20186@163.com

*Contributed equally

Key words: chronic hepatitis B, abdominal obesity, liver fibrosis, non-alcoholic fatty liver, indicators in $\mathrm{CHB}$ patients. For $\mathrm{CHB}$ patients with abdominal obesity and a risk of developing liver fibrosis, priority screening and timely intervention should be provided.

\section{Introduction}

Hepatitis B virus (HBV) infection is one of the major etiologies of chronic liver disease (1). The pathologies secondary to $\mathrm{HBV}$ infection are highly variable, ranging from chronic hepatitis, fibrosis, cirrhosis to hepatocellular carcinoma (HCC). The goal of therapy in patients with chronic hepatitis $\mathrm{B}(\mathrm{CHB})$ is to suppress $\mathrm{HBV}$ reproduction in order to prevent the complications of $\mathrm{HBV}$-associated liver diseases, including necroinflammation, fibrosis, cirrhosis and HCC (1). Hence, patients with $\mathrm{CHB}$ infection require anti-viral therapy and long-term follow-up (2-4). Early identification of CHB infection is important for early diagnosis of liver fibrosis (5).

Another important health burden is obesity. Due to preferences for unhealthy food and decreased physical activity of individuals, obesity has gradually become a new epidemic (6). Abdominal obesity is a type of obesity that is commonly associated with severe metabolic disorders and cardiovascular diseases. An estimated $30 \%$ of adults in Europe and the Americas and $20 \%$ of adults in Asia have abdominal obesity (7-9). Furthermore, an increasing number of patients with CHB infection have developed abdominal obesity $(10,11)$. Previous studies have indicated that abdominal obesity may induce non-alcoholic fatty liver disease (NAFLD), insulin resistance, type 2 diabetes mellitus (T2DM) and even metabolic syndrome (12). A previous study has observed an association between central obesity and advanced liver stiffness in the context of HBV infection (13). Wong et al (14) indicated that coincidental metabolic syndrome increases the risk of liver fibrosis progression in CHB patients, independent viral load and hepatitis activity. Those studies suggest that obesity may have a role in liver fibrosis among patients with CHB. However, the association between abdominal obesity and liver fibrosis in patients with $\mathrm{CHB}$ infection remains to be fully elucidated. In the present study, CHB patients with or without abdominal obesity were enrolled and the prevalence of liver fibrosis in these groups was determined. Indicators of liver fibrosis were explored to provide medical evidence for 
screening and early diagnosis of liver fibrosis in CHB patients with abdominal obesity.

\section{Materials and methods}

Subjects. The present cross-sectional study included a total of $615 \mathrm{CHB}$ patients. Among them, 328 had abdominal obesity and 287 had no abdominal obesity. All patients were enrolled at the Yantai Infectious Disease Hospital and Jining First People's Hospital (Jining, China) between May 2014 and March 2017. The inclusion criteria for patient selection were as follows: i) Chronic HBV infection with the diagnostic criterion of positivity for hepatitis $\mathrm{B}$ surface antigen for $>6$ months ii) Abdominal obesity, determined as a waist circumference of $\geq 90 \mathrm{~cm}$ in males or $\geq 80 \mathrm{~cm}$ in females according to the recommendations of the International Diabetes Federation (15). iii) All patients enrolled received Fibroscan test and were anti-viral treatment-naïve. The exclusion criteria were as follows: i) Infection with hepatitis $\mathrm{C}$ virus or HIV, or other chronic liver diseases, including alcoholic liver disease or cholestatic liver disease. ii) Other serious extra-hepatic diseases, such as chronic heart failure and chronic obstructive pulmonary diseases.

Fibroscan test. The Fibroscan test (Echosens) was performed by two operators trained according to the manufacturer's handbook. A result was considered reliable only if the Fibroscan had at least 10 successful results and the success rate was $>60 \%$, with an inter-quartile median ratio of $<30 \%$. Each patient enrolled required at least 10 successful Fibroscan tests and the median of all tests was used as the liver stiffness value. According to previous studies, a liver stiffness value of $>7.4 \mathrm{kpa}$ was considered to indicate liver cirrhosis and a value of $>10.6 \mathrm{kpa}$ indicated advanced liver cirrhosis according to the METAVIR scoring system (16).

Collection of patient information. Patient information, including demographic data, as well as the results of physical examination and laboratory test, was collected. The demographic information included age, gender, presence of T2DM, history of smoking and drinking. The physical examination data, including body height and weight, as well as waist circumference, were measured and recorded. The blood pressure was also measured after the Fibroscan test. Laboratory tests, including assessment of platelet (PLT), serum aspartate aminotransferase (AST) and alanine aminotransferase (ALT) levels, were performed according to standard procedures. These laboratory results were obtained by standard automated techniques within 14 days of the Fibroscan test.

Blood pressure was measured using a standard mercury sphygmomanometer. All patients were requested to rest for at least $5 \mathrm{~min}$ prior to each measurement. For each patient, at least 3 blood pressure measurements were performed with intervals of $1 \mathrm{~min}$. The blood pressure was determined as the average value of the 3 measurements.

Statistical analysis. Continuous variables were expressed as the mean \pm standard deviation and categorical variables were expressed as numbers and percentages. The $\chi^{2}$ test and Student's t-test were used to detect whether differences between the two groups were statistically significant. Univariate and multivariate logistic regression analyses [forward selection (conditional) model] were used to explore the indicators associated with liver fibrosis, advanced liver fibrosis and liver steatosis in $\mathrm{CHB}$ patients with abdominal obesity. The following cut-off values were used: Age, 43 years; body height, $165 \mathrm{~cm}$; body weight, $67 \mathrm{~kg}$; SBP, $128 \mathrm{mmHg}$, DBP, $84 \mathrm{mmHg}$, ALT, 91 U/1, AST, 58 U/l; and PLT, 242 giga/l. SPSS software (version 13.0; SPSS, Inc.) was used for statistical analysis.

\section{Results}

Demographic and clinical characteristics of patients. A total of 615 patients with $\mathrm{CHB}$ were enrolled in the present study. Among these patients, 287 had abdominal obesity (abdominal obesity group) and 328 were non-abdominal obesity patients (non-abdominal obesity group). The proportion of males in the abdominal obesity group was significantly higher than that in the non-abdominal obesity group. The age, body height and weight, systolic blood pressure (SBP) and diastolic blood pressure (DBP) of patients in the abdominal obesity group were higher than those in the non-abdominal obesity group. The proportions of patients with smoking history, drinking history, T2DM and NAFLD in the non-abdominal obesity group were significantly lower than those in the abdominal obesity group. The serum levels of ALT and AST, and the proportion of patients with hyperlipidemia were comparable between the two groups, as presented in Table I.

Comparison of liver fibrosis between the two groups. The condition of the liver regarding fibrosis was compared between the two groups (Table II). The liver stiffness value in the abdominal obesity group was significantly higher than that in the non-abdominal obesity group $(9.94 \pm 11.59$ vs. $7.47 \pm 7.58$; $\mathrm{P}=0.002$ ). The proportion of patients with liver fibrosis and advanced fibrosis in the abdominal obesity group was significantly higher than that in the non-abdominal obesity group $(\mathrm{P}=0.011)$ according to the METAVIR scores.

Clinical characteristics of abdominal obesity patients with or without liver fibrosis. To further determine the factors associated with liver fibrosis in CHB patients with abdominal obesity, the patients in the abdominal obesity group were stratified into fibrosis and non-fibrosis subgroups. The clinical characteristics of the two subgroups are presented in Table III. The average age and AST levels in patients with fibrosis were significantly higher than those in the non-fibrosis group, while the PLT level was lower in the fibrosis group as compared with that in the non-fibrosis group. In addition, the proportion of patients with a smoking history, alcohol consumption history and diabetes in the fibrosis group was significantly higher than that in the non-fibrosis group.

Indicators associated with fibrosis in CHB patients with abdominal obesity. Univariate and multivariate analyses were performed to explore the indicators associated with fibrosis among CHB patients with abdominal obesity (Table IV). Univariate analysis indicated that $>43$ years, a high AST level, a low PLT level, smoking, alcohol consumption and T2DM were significant indicators associated with liver fibrosis in 
Table I. Baseline demographic and clinical characteristics in the two groups of patients with chronic hepatitis B.

\begin{tabular}{|c|c|c|c|}
\hline \multirow[b]{2}{*}{ Variable } & \multicolumn{2}{|c|}{ Abdominal obesity } & \multirow[b]{2}{*}{ P-value } \\
\hline & Yes $(n=287)$ & No $(n=328)$ & \\
\hline Sex, M/F & $257 / 30$ & $188 / 40$ & $<0.001$ \\
\hline Age (years) & $44.22 \pm 11.79$ & $40.84 \pm 12.44$ & 0.001 \\
\hline Body height (cm) & $168.39 \pm 6.58$ & $163.76 \pm 7.57$ & $<0.001$ \\
\hline Body weight (kg) & $74.49 \pm 10.69$ & $57.35 \pm 7.66$ & $<0.001$ \\
\hline $\mathrm{SBP}(\mathrm{mmHg})$ & $131.92 \pm 12.58$ & $124.94 \pm 12.23$ & $<0.001$ \\
\hline $\mathrm{DBP}(\mathrm{mmHg})$ & $85.29 \pm 8.47$ & $80.77 \pm 8.07$ & $<0.001$ \\
\hline ALT, U/1 & $93.59 \pm 51.61$ & $87.59 \pm 46.11$ & 0.145 \\
\hline AST, U/1 & $58.11 \pm 26.26$ & $58.40 \pm 34.28$ & 0.908 \\
\hline PLT, G/1 & $238.29 \pm 62.92$ & $245.31 \pm 57.74$ & 0.153 \\
\hline HBV DNA (log IU/ml) & $5.25 \pm 3.97$ & $5.57 \pm 3.58$ & 0.294 \\
\hline Smoking, yes $(\%)$ & $128(44.6 \%)$ & $75(22.9 \%)$ & $<0.001$ \\
\hline Alcohol consumption, yes (\%) & $115(40.1 \%)$ & $54(16.5 \%)$ & $<0.001$ \\
\hline T2DM, yes $(\%)$ & $74(25.8 \%)$ & $25(7.6 \%)$ & $<0.001$ \\
\hline NAFLD, yes (\%) & $221(77.0 \%)$ & $113(34.5 \%)$ & $<0.001$ \\
\hline Hyperuricemia, yes (\%) & $70(24.4 \%)$ & $69(21.0 \%)$ & 0.321 \\
\hline Hyperlipidemia, yes (\%) & $68(23.7 \%)$ & $29(8.8 \%)$ & $<0.001$ \\
\hline
\end{tabular}

The normal ranges: ALT, 0-40 U/l; AST, 0-40 U/l; PLT, 100-300 G/l, HBV DNA <500 log IU/ml. Values are expressed as the mean \pm standard deviation, $n$ or percentage. ALT, alanine aminotransferase; AST, aspartate aminotransferase; PLT, platelets; HBV, hepatitis B virus; T2DM, type 2 diabetes mellitus; NAFLD, non-alcoholic fatty liver disease; SBP, systolic blood pressure; DBP, diastolic blood pressure; G/l, giga per liter.

Table II. Parameters of liver fibrosis and advanced fibrosis in the two groups of patients with chronic hepatitis B.

\begin{tabular}{lcc}
\hline & \multicolumn{2}{c}{ Abdominal obesity } \\
\cline { 2 - 3 } Variable & Yes (n=287) & No (n=328) \\
\hline METAVIR score & $175(60.9 \%)$ & $237(72.3 \%)$ \\
F0 & $46(16.1 \%)$ & $42(12.8 \%)$ \\
F1-F2 & $66(23.0 \%)$ & $49(14.9 \%)$ \\
PF2 & $9.94 \pm 11.59$ & $7.47 \pm 7.58$ \\
Liver stiffness value (kpa) & & 0.009 \\
\hline
\end{tabular}

Values are expressed as the mean \pm standard deviation, $\mathrm{n}$ or percentage.

CHB patients with abdominal obesity. Multivariate analysis suggested that a high AST [(odds ratio $(\mathrm{OR})=1.003 ; \mathrm{P}<0.001]$, a low PLT $(\mathrm{OR}=0.991 ; \mathrm{P}<0.001)$, smoking $(\mathrm{OR}=2.002 ; \mathrm{P}=0.019)$ and T2DM $(\mathrm{OR}=2.047 ; \mathrm{P}=0.029)$ were independent indicators associated with liver fibrosis in $\mathrm{CHB}$ patients with abdominal obesity.

Indicators associated with advanced fibrosis in CHB patients with abdominal obesity. Factors associated with advanced liver fibrosis in $\mathrm{CHB}$ patients with abdominal obesity were then determined (Table V). Univariate analysis suggested that an older age, a high AST level, a low PLT level, smoking, alcohol consumption and T2DM were significant indicators for advanced fibrosis in CHB patients with abdominal obesity. Multivariate analysis indicated that only advanced
AST $(\mathrm{OR}=1.024 ; \mathrm{P}<0.001)$, alcohol consumption $(\mathrm{OR}=1.994$; $\mathrm{P}=0.032)$ and diabetes $(\mathrm{OR}=1.977 ; \mathrm{P}=0.045)$ were independent indicators for advanced liver fibrosis.

Indicators associated with liver steatosis in CHB patients with abdominal obesity. Indicators associated with liver steatosis among $\mathrm{CHB}$ patients with abdominal obesity were then determined (Table VI). Univariate analysis suggested that a high body weight, SBP and DBP and a high AST level were significant indicators for liver steatosis among $\mathrm{CHB}$ patients with abdominal obesity. Subsequent multivariate analysis indicated that only a high body weight $(\mathrm{OR}=1.113$; $\mathrm{P}<0.001)$ and high $\mathrm{DBP}(\mathrm{OR}=1.079 ; \mathrm{P}=0.002)$ were independent indicators for liver steatosis in $\mathrm{CHB}$ patients with abdominal obesity. 
Table III. Characteristics of subjects with chronic hepatitis B and abdominal obesity with or without liver fibrosis.

Fibrosis

\begin{tabular}{|c|c|c|c|}
\hline \multirow[b]{2}{*}{ Variable } & & \multirow[b]{2}{*}{ P-value } \\
\hline & Yes $(n=112)$ & No $(n=175)$ & \\
\hline $\operatorname{Sex}, M / F$ & $99 / 13$ & $158 / 17$ & 0.609 \\
\hline Age (years) & $45.99 \pm 12.29$ & $43.09 \pm 11.37$ & 0.042 \\
\hline Body height (cm) & $168.09 \pm 6.93$ & $168.58 \pm 6.36$ & 0.544 \\
\hline Body weight (kg) & $75.33 \pm 12.34$ & $73.95 \pm 9.49$ & 0.288 \\
\hline $\mathrm{SBP}(\mathrm{mmHg})$ & $132.76 \pm 12.91$ & $130.59 \pm 11.98$ & 0.158 \\
\hline DBP (mmHg) & $85.9 \pm 8.61$ & $84.29 \pm 8.17$ & 0.111 \\
\hline ALT, U/1 & $87.42 \pm 38.52$ & $87.69 \pm 50.44$ & 0.963 \\
\hline $\mathrm{AST}, \mathrm{U} / 1$ & $69.27 \pm 27.61$ & $50.98 \pm 22.71$ & $<0.001$ \\
\hline PLT, G/1 & $216.16 \pm 72.22$ & $252.25 \pm 51.84$ & $<0.001$ \\
\hline HBV DNA $(\log I U / m l)$ & $6.01 \pm 3.45$ & $5.27 \pm 3.12$ & 0.061 \\
\hline Smoking, yes/no & $65(58.0 \%)$ & $63(25.8 \%)$ & $<0.001$ \\
\hline Alcohol consumption, yes/no & $53(47.3 \%)$ & $62(35.4 \%)$ & 0.045 \\
\hline T2DM, yes/no & $41(36.6 \%)$ & $33(18.9 \%)$ & 0.001 \\
\hline NAFLD, yes/no & $81(72.3 \%)$ & $140(80.0 \%)$ & 0.109 \\
\hline Hyperuricemia, yes/no & $23(20.5 \%)$ & $47(26.9 \%)$ & 0.224 \\
\hline Hyperlipidemia, yes/no & $28(25.0 \%)$ & $40(22.9 \%)$ & 0.677 \\
\hline
\end{tabular}

Values are expressed as the mean \pm standard deviation, $\mathrm{n}$ or percentage. ALT, alanine aminotransferase; AST, aspartate aminotransferase; PLT, platelets; HBV, hepatitis B virus; T2DM, type 2 diabetes mellitus; NAFLD, non-alcoholic fatty liver disease; SBP, systolic blood pressure; DBP, diastolic blood pressure; G/1, giga per liter.

\section{Discussion}

An estimated 240 million individuals worldwide have CHB (1). Although the clinical management of HBV infection and CHB, including anti-viral therapy, have been widely established, CHB infection remains one of the major etiologies of liver fibrosis and cirrhosis (17-20). Cirrhosis is characterized by diffuse fibrosis and liver tissue abnormalities, including hepatocyte necrosis and regenerative liver nodules (21). Liver cirrhosis is a indicator for end-stage liver disease, including decompensated liver disease, and HCC (22-24). Therefore, early diagnosis of liver fibrosis is important for the clinical management of CHB patients. Liver biopsy is the gold standard for assessing hepatic fibrosis and steatosis. However, liver biopsy is associated with a risk of complications. The rate of complications is $\sim 0.5 \%$ and the fatality rate is $\sim 0.05 \%$ (5). In clinical practice, it is difficult to perform liver biopsy in a large population (11). Exploring the indicators for liver fibrosis in patients with $\mathrm{CHB}$ and monitoring a high-risk subpopulation is a more suitable clinical strategy.

Abdominal obesity has been confirmed to be associated with diabetes, hypertension and NAFL (10). The number of individuals with abdominal obesity has been on the rise, and a significant proportion of $\mathrm{CHB}$ patients have abdominal obesity. Although it has been demonstrated that diabetes may lead to liver fibrosis (25), the impact of abdominal obesity on patients with CHB-associated liver fibrosis has not been explored. The present study revealed that CHB patients with abdominal obesity had a significantly higher liver stiffness value than those without abdominal obesity. Furthermore, in the abdominal obesity group, the proportion of patients with liver fibrosis and advanced liver fibrosis were higher compared with those in the non-abdominal obesity group. These results suggest that for CHB patients, abdominal obesity may exacerbate the progression of liver fibrosis. Therefore, screening for liver fibrosis in $\mathrm{CHB}$ patients with abdominal obesity and timely intervention are of great importance.

The present study confirms that high AST and low PLT levels are independent indicators for hepatic fibrosis in $\mathrm{CHB}$ patients with abdominal obesity. This result is consistent with that of a previous study where AST and PLT were regarded as indicators of liver fibrosis in CHB patients (26). The present study also suggested that smoking promotes liver fibrosis in CHB patients with abdominal obesity. Previous studies have indicated that smoking may promote liver fibrosis in patients with primary biliary cirrhosis, but the exact mechanism has remained elusive (27). In vitro experiments have suggested that smoking may induce activation of hepatocyte apoptosis by releasing metalloproteinases and promoting Fas expression $(28,29)$. In addition, smoking may stimulate hepatic stellate cells through oxidative stress, and induce the expression of interleukin (IL)-1, IL- 6 and tumor necrosis factor- $\alpha$, thereby promoting the progress of liver fibrosis (30). The present study confirmed that smoking is an independent indicator for hepatic fibrosis in CHB patients with abdominal obesity. It is therefore indicated that smoking cessation is beneficial for $\mathrm{CHB}$ patients, particularly for those with abdominal obesity.

The present study suggested that alcohol consumption is an independent indicator for advanced liver fibrosis in 
Table IV. Risk factors associated with fibrosis in chronic hepatitis B with abdominal obesity.

\begin{tabular}{|c|c|c|c|c|c|c|}
\hline \multirow[b]{2}{*}{ Variable } & \multicolumn{3}{|c|}{ Univariate analysis } & \multicolumn{3}{|c|}{ Multivariate analysis } \\
\hline & OR & $95 \% \mathrm{CI}$ & P-value & OR & $95 \% \mathrm{CI}$ & P-value \\
\hline Sex, male vs. female & 0.819 & $0.381-1.760$ & 0.610 & & & \\
\hline Age, years & 1.021 & $1.001-1.042$ & 0.043 & & & \\
\hline Body height, cm & 0.989 & $0.954-1.025$ & 0.542 & & & \\
\hline Body weight, kg & 1.012 & $0.990-1.035$ & 0.291 & & & \\
\hline $\mathrm{SBP}, \mathrm{mmHg}$ & 0.986 & $0.967-1.005$ & 0.159 & & & \\
\hline DBP, mmHg & 0.977 & $0.949-1.005$ & 0.112 & & & \\
\hline ALT, U/1 & 1.000 & $0.995-1.005$ & 0.963 & & & \\
\hline AST, U/l & 1.032 & $1.020-1.043$ & $<0.001$ & 1.033 & $1.019-1.047$ & $<0.001$ \\
\hline PLT, G/1 & 0.990 & $0.986-0.994$ & $<0.001$ & 0.991 & $0.987-0.996$ & $<0.001$ \\
\hline Smoking, no vs. yes & 2.459 & $1.512-3.997$ & $<0.001$ & 2.002 & $1.123-3.568$ & 0.019 \\
\hline Alcohol consumption, no vs. yes & 1.637 & $1.010-2.655$ & 0.046 & & & \\
\hline T2DM, no vs. yes & 2.485 & $1.448-4.263$ & 0.001 & 2.047 & $1.075-3.900$ & 0.029 \\
\hline NAFLD, no vs. yes & 0.635 & $0.363-1.109$ & 0.11 & & & \\
\hline Hyperuricemia, no vs. yes & 0.704 & $0.399-1.241$ & 0.225 & & & \\
\hline Hyperlipidemia, no vs. yes & 1.125 & $0.646-1.959$ & 0.677 & & & \\
\hline
\end{tabular}

OR, odds ratio; ALT, alanine aminotransferase; AST, aspartate aminotransferase; PLT, platelets; HBV, hepatitis B virus; T2DM, type 2 diabetes mellitus; NAFLD, non-alcoholic fatty liver disease; SBP, systolic blood pressure; DBP, diastolic blood pressure; G/l, giga per liter.

Table V. Risk factors for advanced fibrosis in chronic hepatitis B with abdominal obesity.

\begin{tabular}{|c|c|c|c|c|c|c|}
\hline \multirow[b]{2}{*}{ Variable } & \multicolumn{3}{|c|}{ Univariate analysis } & \multicolumn{3}{|c|}{ Multivariate analysis } \\
\hline & OR & $95 \% \mathrm{CI}$ & $\mathrm{P}$-value & OR & $95 \% \mathrm{CI}$ & P-value \\
\hline Sex, male vs. female & 0.802 & $0.339-1.895$ & 0.614 & & & \\
\hline Age, years & 1.026 & $1.003-1.050$ & 0.029 & & & \\
\hline Body height, $\mathrm{cm}$ & 1.000 & 0.959-1.042 & 0.983 & & & \\
\hline Body weight, kg & 1.017 & $0.992-1.042$ & 0.176 & & & \\
\hline $\mathrm{SBP}, \mathrm{mmHg}$ & 0.981 & $0.959-1.004$ & 0.113 & & & \\
\hline $\mathrm{DBP}, \mathrm{mmHg}$ & 0.97 & $0.937-1.003$ & 0.076 & & & \\
\hline ALT, U/1 & 0.997 & $0.990-1.003$ & 0.310 & & & \\
\hline $\mathrm{AST}, \mathrm{U} / 1$ & 1.017 & $1.005-1.028$ & 0.004 & 1.024 & $1.011-1.038$ & $<0.001$ \\
\hline PLT, G/1 & 0.994 & 0.989-0.999 & 0.010 & & & \\
\hline HBV DNA, $\log$ IU/ml & 1.096 & $0.948-1.126$ & 0.391 & & & \\
\hline Smoking, no vs. yes & 1.823 & $1.046-3.175$ & 0.034 & & & \\
\hline Alcohol consumption, no vs. yes & 1.836 & 1.054-3.199 & 0.032 & 1.994 & $1.061-3.747$ & 0.032 \\
\hline T2DM, no vs. yes & 2.140 & $1.186-3.861$ & 0.011 & 1.977 & $1.016-3.850$ & 0.045 \\
\hline NAFLD, no vs. yes & 0.591 & $0.319-1.098$ & 0.096 & & & \\
\hline Hyperuricemia, no vs. yes & 1.100 & $0.584-2.069$ & 0.768 & & & \\
\hline Hyperlipidemia, no vs. yes & 1.419 & $0.763-2.638$ & 0.269 & & & \\
\hline
\end{tabular}

OR, odds ratio; ALT, alanine aminotransferase; AST, aspartate aminotransferase; PLT, platelets; HBV, hepatitis B virus; T2DM, type 2 diabetes mellitus; NAFLD, non-alcoholic fatty liver disease; SBP, systolic blood pressure; DBP, diastolic blood pressure; G/1, giga per liter.

CHB patients with abdominal obesity. Previous studies have demonstrated that alcohol is an inducer of hepatocyte apoptosis (31). With increases in alcohol intake, hepatocyte apoptosis increases significantly. Alcohol is transformed to acetaldehyde in hepatocytes and directly damages the hepatocyte membrane (32). The results of the present study confirm that alcohol consumption is an independent indicator for advanced hepatic fibrosis in CHB patients with abdominal 
Table VI. Risk factors for liver steatosis in chronic hepatitis B patients with abdominal obesity.

\begin{tabular}{|c|c|c|c|c|c|c|}
\hline \multirow[b]{2}{*}{ Variable } & \multicolumn{3}{|c|}{ Univariate analysis } & \multicolumn{3}{|c|}{ Multivariate analysis } \\
\hline & OR & $95 \% \mathrm{CI}$ & P-value & OR & $95 \% \mathrm{CI}$ & P-value \\
\hline Sex, male vs. female & 0.492 & $0.165-1.465$ & 0.202 & & & \\
\hline Age, years & 0.993 & $0.970-1.016$ & 0.561 & & & \\
\hline Body height, $\mathrm{cm}$ & 0.991 & $0.950-1.034$ & 0.693 & & & \\
\hline Body weight, $\mathrm{kg}$ & 1.059 & $1.023-1.096$ & 0.001 & 1.113 & $1.047-1.183$ & 0.001 \\
\hline $\mathrm{SBP}, \mathrm{mmHg}$ & 1.031 & $1.006-1.056$ & 0.016 & & & \\
\hline $\mathrm{DBP}, \mathrm{mmHg}$ & 1.067 & $1.028-1.107$ & 0.001 & 1.079 & $1.029-1.132$ & 0.002 \\
\hline ALT, U/1 & 0.999 & $0.992-1.005$ & 0.652 & & & \\
\hline AST, U/l & 1.013 & $1.002-1.025$ & 0.025 & & & \\
\hline PLT, G/l & 1.000 & $0.996-1.005$ & 0.949 & & & \\
\hline HBV DNA, $\log I U / m l$ & 0.941 & $0.844-1.239$ & 0.628 & & & \\
\hline Smoking, no vs. yes & 0.989 & $0.567-1.725$ & 0.969 & & & \\
\hline Alcohol consumption, no vs. yes & 1.278 & $0.720-2.271$ & 0.402 & & & \\
\hline T2DM, no vs. yes & 0.728 & $0.395-1.338$ & 0.306 & & & \\
\hline Hyperuricemia, no vs. yes & 1.236 & $0.636-2.403$ & 0.531 & & & \\
\hline Hyperlipidemia, no vs. yes & 1.706 & $0.835-3.488$ & 0.143 & & & \\
\hline
\end{tabular}

OR, odds ratio; ALT, alanine aminotransferase; AST, aspartate aminotransferase; PLT, platelets; HBV, hepatitis B virus; T2DM, type 2 diabetes mellitus; NAFLD, non-alcoholic fatty liver disease; SBP, systolic blood pressure; DBP, diastolic blood pressure; G/l, giga per liter.

obesity. Timely intervention in the form of abstinence of CHB patients with abdominal obesity may help to delay the progression of the disease and improve the long-term prognosis.

It has been suggested that metabolic syndrome is an indicator of advanced liver fibrosis and cirrhosis independent of viral load in CHB patients (14). Metabolic syndrome, comprising T2DM, hypertension, central obesity and dyslipidaemia, is increasingly prevalent worldwide, particularly in Asia $(10,33)$. A dose-response association between the number of components of metabolic syndrome and the risk of advanced fibrosis was observed in CHB patients (10). Adipokines may have a role in liver fibrosis in CHB patients. Therefore, the dynamic and interactive effect of viral and metabolic factors on liver fibrosis warrants further investigation in a prospective study.

The present study indicated that CHB patients with a high body weight and high DBP are more prone to developing liver steatosis in the abdominal obesity group. NAFL is the liver manifestation of metabolic syndrome. A previous study has indicated that CHB combined with NAFLD is a indicator for poor response to entercavir (34). Chan et al (35) revealed that in CHB patients with NAFL, the risk of liver cancer was increased by 7.3 -fold. Based on the results of the present study, CHB patients with abdominal obesity should be screened for NAFL, particularly those that are overweight or have a high DBP. Although the disease course is an important factor affecting disease progression, it was not analyzed in the present study. The course of the disease was not included as a variable for analysis in the present study for the following reasons: Certain patients may lack knowledge regarding their disease course or it is not accurately reported by them. Furthermore, in numerous cases, infection may have occurred during childhood or even as mother-to-child transmission, and is then only detected in adulthood.

However, based on previous studies, liver fibrosis may be diagnosed if the liver stiffness value is $>7.4 \mathrm{kpa}$, and advanced liver fibrosis is indicated if the value is $>10.6 \mathrm{kpa}$ in patients with normal bilirubin and ALT $<2 \mathrm{x}$ upper limit of normal (16). For patients with a higher ALT, the cutoff value of the liver stiffness value should be set higher. However, the use of a higher cut-off point leads to false-negative results. Since the aim of the present study was to identify a subpopulation with a risk of liver fibrosis, the cut-off value was set lower, allowing for better identification of liver fibrosis.

The present study had certain limitations. First, the sample size of the study was relatively small. Furthermore, the data included in the present study were from a single center. Some patients with other types of obesity, such as general obesity, may be classified as patients with abdominal obesity, which is another limitation of this study. The use of height and weight, rather than BMI, was also a limitation of the current study. Given the cross-sectional nature of the present study, propensity score matching or another prospective study should be performed to corroborate the conclusions. Multi-center clinical studies are warranted to confirm the present results, providing a strategy for screening and early diagnosis of liver fibrosis in CHB patients with abdominal obesity. The impact of these factors on the future development of fibrosis and steatosis is need to be assessed in a future study.

In conclusion, to the best of our knowledge, the present study was the first to demonstrate that abdominal obesity is associated with liver fibrosis in CHB patients. For CHB patients with abdominal obesity, priority screening and timely 
intervention should be provided if those patients have indicators for liver fibrosis.

\section{Acknowledgements}

Not applicable.

\section{Funding}

No funding was received.

\section{Availability of data and materials}

The datasets used and/or analyzed during the present study are available from the corresponding author on reasonable request.

\section{Authors' contributions}

JS drafted the manuscript. JS and YL recorded and analyzed the data of patients enrolled. XS and YL conducted the statistical analysis. DZ and LF provided substantial contributions in data acquisition. The final version was read and approved by all authors.

\section{Ethical approval and consent to participate}

The Institutional Review Board of Yantai Infectious Disease Hospital (Yantai, China) approved the present study. Each of the enrolled patients provided written informed consent.

\section{Patient consent for publication}

Not applicable.

\section{Competing interests}

The authors declare that they have no competing interests.

\section{References}

1. Sarin SK, Kumar M, Lau GK, Abbas Z, Chan HL, Chen CJ, Chen DS, Chen HL, Chen PJ, Chien RN, et al: Asian-pacific clinical practice guidelines on the management of hepatitis B: A 2015 update. Hepatol Int 10: 1-98, 2016.

2. Xue X, Cai S, Ou H, Zheng C and Wu X: Health-related quality of life in patients with chronic hepatitis B during antiviral treatment and off-treatment. Patient Prefer Adherence 11: 85-93, 2017.

3. Wu X, Cai S, Li Z, Zheng C, Xue X, Zeng J and Peng J: Potential effects of telbivudine and entecavir on renal function: A systematic review and meta-analysis. Virol J 13: 64, 2016.

4. Peng J, Yin J, Cai S, Yu T and Zhong C: Factors associated with adherence to nucleos(t)ide analogs in chronic hepatitis B patients: Results from a 1 year follow-up study. Patient Prefer Adherence 9: 41-45, 2015.

5. Lok AS and McMahon BJ: Chronic hepatitis b: Update 2009. Hepatology 50: 661-662, 2009.

6. Gutierrez-Fisac JL, Guallar-Castillon P, Leon-Munoz LM, Graciani A, Banegas JR and Rodriguez-Artalejo F: Prevalence of general and abdominal obesity in the adult population of Spain, 2008-2010: The ENRICA study. Obes Rev 13: 388-392, 2012.

7. Zhai Y, Fang HY, Yu WT, Yu DM, Zhao LY, Liang XF and Zhao WH: Changes in waist circumference and abdominal obesity among chinese adults over a ten-year period. Biomed Environ Sci 30: 315-322, 2017.
8. Suder A, Janusz M, Jagielski P, Głodzik J, Pałka T, Cisoń T and Pilch W: Prevalence and risk factors of abdominal obesity in polish rural children. Homo 66: 357-368, 2015.

9. Lim J and Park HS: Trends in the prevalence of underweight, obesity, abdominal obesity and their related lifestyle factors in korean young adults, 1998-2012. Obes Res Clin Pract 12: 358-364, 2017.

10. Cai S, Ou Z, Liu D, Liu L, Liu Y, Wu X, Yu T and Peng J: Risk factors associated with liver steatosis and fibrosis in chronic hepatitis B patient with component of metabolic syndrome. United European Gastroenterol J 6: 558-566, 2018.

11. Ou H, Cai S, Liu Y, Xia M and Peng J: A noninvasive diagnostic model to assess nonalcoholic hepatic steatosis in patients with chronic hepatitis B. Therap Adv Gastroenterol 10: 207-217, 2017.

12. European Association for the Study of the Liver (EASL) European Association for the Study of Diabetes (EASD) and European Association for the Study of Obesity (EASO): EASL-EASD-EASO clinical practice guidelines for the management of non-alcoholic fatty liver disease. J Hepatol 64: 1388-1402, 2016.

13. Besharat S, Poustchi H, Mohamadkhani A, Roshandel G Freedman ND, Merat S and Malekzadeh R: Central obesity and advanced liver stiffness in hepatitis b: Result from golestan hepatitis b cohort study. Arch Iran Med 18: 562-566, 2015.

14. Wong L, Chan HL, Yu Z, Chan AW, Choi PC, Chim AM, Chan HY, Tse $\mathrm{CH}$ and Wong VW: Coincidental metabolic syndrome increases the risk of liver fibrosis progression in patients with chronic hepatitis B-a prospective cohort study with paired transient elastography examinations. Aliment Pharmacol Ther 39: 883-893, 2014.

15. Alberti KG, Zimmet P and Shaw J: The metabolic syndrome-a new worldwide definition. Lancet 366: 1059-1062, 2005.

16. Chen YP, Liang XE, Zhang Q, Peng J, Zhu YF, Wen WQ and Hou JL: Larger biopsies evaluation of transient elastography for detecting advanced fibrosis in patients with compensated chronic hepatitis B. J Gastroenterol Hepatol 27: 1219-1226, 2012.

17. Cai S, Cao J, Yu T, Xia M and Peng J: Effectiveness of entecavir or telbivudine therapy in patients with chronic hepatitis B virus infection pre-treated with interferon compared with de novo therapy with entecavir and telbivudine. Medicine (Baltimore) 96: e7021, 2017.

18. Xue X and Cai S: Comment on 'assessment of liver stiffness in pediatric fontan patients using transient elastography'. Can J Gastroenterol Hepatol 2016: 9343960, 2016.

19. Cai S, Yu T, Jiang Y, Zhang Y, Lv F and Peng J: Comparison of entecavir monotherapy and de novo lamivudine and adefovir combination therapy in HBeAg-positive chronic hepatitis B with high viral load: 48 week result. Clin Exp Med 16: 429-436, 2016.

20. Cai SH, Lv FF, Zhang YH, Jiang YG and Peng J: Dynamic comparison between daan real-time PCR and cobas taqman for quantification of HBV DNA levels in patients with Chb. Bmc Infect Dis 14: 85, 2014.

21. Zeng J, Cai S, Liu J, Xue X, Wu X and Zheng C: Dynamic changes in liver stiffness measured by transient elastography predict clinical outcomes among patients with chronic hepatitis b. J Ultrasound Med 36: 261-268, 2017.

22. Cai SH, Lu SX, Liu LL, Zhang CZ and Yun JP: Increased expression of hepatocyte nuclear factor 4 alpha transcribed by promoter 2 indicates a poor prognosis in hepatocellular carcinoma. Therap Adv Gastroenterol 10: 761-771, 2017.

23. Chen SL, Liu LL, Lu SX, Luo RZ, Wang CH, Wang H, Cai SH, Yang X, Xie D, Zhang CZ and Yun JP: HBx-mediated decrease of AIM2 contributes to hepatocellular carcinoma metastasis. Mol Oncol 11: 1225-1240, 2017.

24. Cai S, Li Z, Yu T, Xia M and Peng J: Serum hepatitis B core antibody levels predict $\mathrm{HBeAg}$ seroconversion in chronic hepatitis $\mathrm{B}$ patients with high viral load treated with nucleos(t)ide analogs. Infect Drug Resist 11: 469-477, 2018.

25. Roulot D, Roudot-Thoraval F, Nkontchou G, Kouacou N, Costes JL, Elourimi G, Le Clesiau H, Ziol M and Beaugrand M: Concomitant screening for liver fibrosis and steatosis in french type 2 diabetic patients using Fibroscan. Liver Int 37: 1897-1906, 2017.

26. Peng J, Cai S, Yu T, Chen Y, Zhu Y and Sun J: Aspartate aminotransferase to platelet ratio index - a reliable predictor of therapeutic efficacy and improvement of Ishak score in chronic hepatitis B patients treated with nucleoside analogues. Scand J Clin Lab Invest 76: 133-142, 2016.

27. Corpechot C, Gaouar F, Chretien Y, Johanet C, Chazouilleres O and Poupon R: Smoking as an independent risk factor of liver fibrosis in primary biliary cirrhosis. J Hepatol 56: 218-224, 2012. 
28. van der Vaart H, Postma DS, Timens W and Ten HN: Acute effects of cigarette smoke on inflammation and oxidative stress: A review. Thorax 59: 713-721, 2004.

29. Bijl M, Horst G, Limburg PC and Kallenberg CG: Effects of smoking on activation markers, Fas expression and apoptosis of peripheral blood lymphocytes. Eur J Clin Invest 31: 550-553, 2001.

30. Zeidel A, Beilin B, Yardeni I, Mayburd E, Smirnov G and Bessler H: Immune response in asymptomatic smokers. Acta Anaesthesiol Scand 46: 959-964, 2002.

31. Ma Z, Hou T, Shi W, Liu W and He H: Inhibition of hepatocyte apoptosis: An important mechanism of corn peptides attenuating liver injury induced by ethanol. Int J Mol Sci 16: 22062-22080, 2015.

32. Kim WH, Hong F, Jaruga B, Hu Z, Fan S, Liang TJ and Gao B: Additive activation of hepatic NF-kappaB by ethanol and hepatitis B protein $\mathrm{X}(\mathrm{HBX})$ or $\mathrm{HCV}$ core protein: Involvement of TNF-alpha receptor 1-independent and -dependent mechanisms. FASEB J 15: 2551-2553, 2001.
33. Xiao YB, Cai SH, Liu LL, Yang X and Yun JP: Decreased expression of peroxisome proliferator-activated receptor alpha indicates unfavorable outcomes in hepatocellular carcinoma. Cancer Manag Res 10: 1781-1789, 2018.

34. Jin X, Chen YP, Yang YD, Li YM, Zheng L and Xu CQ: Association between hepatic steatosis and entecavir treatment failure in Chinese patients with chronic hepatitis B. PLoS One 7: e34198, 2012.

35. Chan AW, Wong GL, Chan HY, Tong JH, Yu YH, Choi PC, Chan HL, To KF and Wong VW: Concurrent fatty liver increases risk of hepatocellular carcinoma among patients with chronic hepatitis B. J Gastroenterol Hepatol 32: 667-676, 2017. 